\title{
Cytogenetic study on domestic pig (Sus scrofa)
}

\author{
C. C. LIN and R. B. CHURCH \\ Division of Pediatrics and Medical Biochemistry \\ The University of Calgary, \\ Calgary, Alberta, Canada $\mathrm{T}_{2} \mathrm{~N}_{\mathrm{I}} \mathrm{N}_{4}$
}

Chromosome analysis using several banding techniques and Ag-As staining techniques has been performed on domestic pig. Attempts were made on the cytological identification of the $\mathrm{X}$-chromosome, G-C rich centromeric regions and the location of ribosomal cistrons. A nomenclature for banded karyotype of this species was also presented.

\section{Ghromosome Aberrations in Pigs after Vaccination with living Vaccine against Swine Fever}

\author{
I. LOJDA and J. RUBES
}

\author{
Veterinary Research Institute, Brno (Tchekoslovaquie)
}

To protect pigs from swine fever, various vaccines have been employed. Good protective effects have been reported for living lapinized vaccine.

It is well established that infection of pregnant sows with swine fever virus produces various malformations of the offspring as well as chromosome mutations.

In the present study 30 pigs, weighing from 30 to $40 \mathrm{~kg}$, were immunized with various batches of living lapinized vaccine against swine fever and 30 pigs were left untreated to serve as controls. The results suggest that even the attenuated virus produces various kinds of chromosome changes. In the experimental pigs the incidence of structural chromosome changes was almost 9 times higher and that of polyploidy about 6 times higher than in the controls. The structural changes consisted of chromosome and chromatid breaks that gave rise to further aberrations.

\section{C-banding in sheep with balanced homolog interchromosomal translocation}

\author{
B. GLAHN-LUFT and R. WASSMUTH \\ Institut für Tierzucht und Haustiergenetik \\ Justus Liebig Universität \\ Bismarkstr. I6, Giessen, 63000 (R.F.A.)
}

The $\mathrm{C}$ - banding- technique is demonstrated in sheep. The comparison of heterozygous, and homozygous translocation animals and a normal karyotype shows a big $\mathrm{C}$ - band in the deletion chromosomes, while a weak $\mathrm{C}$ - band can be seen in the translocation chromosomes. In course of the length the translocation chromosome can be ordered in pair 4-9. Here we have in sheep with the karyotype $2 n=54$, XY or XX no pair with weakly stained C- bands in the autosomes. The descendant with the karyotype $2 n=54, \mathrm{XY}, t\left(1 p^{-} ; 24 q^{\dagger}\right) 2$ can be well identified by $\mathrm{C}$ - banding.

\section{Nucleolus Organisers in Chromosomes of some Domestic Animals}

\author{
P. S. SYSA, Henni HOHN and Imild SCHMIDT \\ Dept. Institut für Zuchthygiene und Veterinarmedizinische Genetik \\ Holmanstrasse IO, Giessen, 63 (R.F.A.)
}

A method of visualizing chromosome $\mathrm{N}$ bands by treatment with $\mathrm{NaH}_{2} \mathrm{PO}_{4}$ at $96{ }^{\circ} \mathrm{C}$ was applied to define the location of nucleolus organisers in chromosomes of cattle, goat, sheep, horse, pig and cat. In the species studied the $\mathbf{N}$ bands were clearly located in certain typical 
regions of chromosomes such as the centromere and telomere in cattle, goat and horse; centromere and "secondary constriction" in pig; satellite in cat. However, polymorphism in occurrence of $\mathrm{N}$ bands was observed.

Influence of the $\mathrm{NaH}_{2} \mathrm{PO}_{4}$ incubation on the discovering of $\mathrm{N}$ bands in the fresh and old slides was observed. Staining procedure for $\mathrm{C}$ banding has been used for the comparison of results.

\title{
Frequency and source of chromosome abnormalities in chick embryos
}

\author{
N. S. FECHHEIMER
}

\author{
Dairy Science Department, The Ohio State University, \\ Columbus, Ohio 43210 (U.S.A.)
}

In a series of nine studies, 8258 chicken embryos have been examined for the occurrence of chromosomal abnormalities. Eggs were incubated for only i 6 to 18 hours before the embryos, in the primitive streak stage, were removed. Preparations for karyological examination were made from cell suspensions of each embryo. The overall incidence of embryos with aberrations was 49 p. I, 000 but there were large and significant differences between stocks of broilertype (BR) and Leghorn-type (LG). BR contained IO2 p. I ooo, LG contained I4.I p. rooo and embryos from crosses between the two contained $46 \mathrm{p}$. Iooo. The most frequently occuring types of abnormalities were haploidy (including haploid-diploid chimerics), polyploidy, diploid-tetraploid mosaics and pure aneuploidy. Their incidences per $\mathrm{I}, 000$ in $B L, L G$ and crosses respectively were 54, 3, I9 for haploidy; 21, 6, 9 for polyploidy; I 6, I, 5 for diploid-tetraploid mosaics; 6,3 , and 9 for aneuploidy. The parental source of each of the primary types of abnormialities has been determined and the place in the reproductive cycle where errors occur giving rise to each has been established. 